\title{
Advanced Control Strategies for Stability Improvement of Natural Field Orientation
}

\author{
G. Mirzaeva* and A. Rojas ${ }^{\dagger}$ \\ School of Electrical Engineering and Computer Science \\ University of Newcastle, NSW, Australia, 2308 \\ Email: *Galina.Mirzaeva@newcastle.edu.au, †Alejandro.Rojas@newcastle.edu.au
}

\begin{abstract}
Natural Field Orientation (NFO) is a patented control strategy for induction machines that was first proposed in 1980's. In recent work by R.E.Betz and G.Mirzaeva it was found that the NFO control algorithm has a stability problem in regeneration mode. The authors proposed an augmentation to the basic algorithm in the form of an auxiliary feedback in the angular velocity estimation. This augmentation resulted in improvement of the NFO performance in regeneration, however, limited to relatively small torque magnitudes and slow dynamics in regeneration. It was expected that flexible control of the auxiliary feedback gain and / or application of a PI block in the auxiliary feedback may be beneficial for further performance improvement of the NFO algorithm.

This paper develops a full dynamic model of the NFO controlled system and presents results of its linearised stability analysis. Based on the above, it proposes some more advanced versions of the control over the parameters of the auxiliary feedback. The paper further discusses advantages and limitations of the proposed modifications of the NFO algorithm. To support the discussion, it includes results of simulations and experiments obtained for a test induction machine.
\end{abstract}

\section{INTRODUCTION}

Natural Field Orientation (NFO) is a patented control strategy proposed in ( [1], [2]). It can be seen as a version of Stator Flux Orientation (SFO) control where:

- calculations are performed in the reference frame aligned with the stator flux vector (space vectors defined in this frame will be denoted by the index " $\psi_{s}$ ") ;

- estimation of the stator flux vector position is based on the "voltage model", i.e. on the back emf estimation using the stator voltage equation as in:

$$
\underline{e}_{s \psi_{s}}=\underline{u}_{s \psi_{s}}-R_{s} \underline{i}_{s \psi_{s}}=\frac{d\left|\psi_{s}\right|}{d t}+j \omega_{m s}\left|\psi_{s}\right|
$$

where $\underline{e}_{s \psi_{s}}$ is stator back emf vector; $\left|\psi_{s}\right|$ is the magnitude of the stator flux vector $\underline{\psi}_{s} ; R_{s}$ is stator resistance; $\omega_{m s}$ is angular velocity of the stator flux vector $\underline{\psi}_{s}$.

- the magnitude of the stator flux vector is not obtained by integration but is assumed to be equal to its reference value.
The last point can be explained as follows. Splitting the stator voltage equation (1) into real and imaginary parts yields:

$$
\begin{aligned}
& e_{s x}=u_{s x}-R_{s} i_{s x}=\frac{d\left|\psi_{s}\right|}{d t}=L_{m} \frac{d\left|i_{m s}\right|}{d t} \\
& e_{s y}=u_{s y}-R_{s} i_{s y}=\omega_{m s}\left|\psi_{s}\right|=\omega_{m s} L_{m}\left|i_{m s}\right|
\end{aligned}
$$

where $x$-axis is aligned with the vector $\underline{\psi}_{s}$ and $y$-axis is in quadrature to it; $L_{m}$ is magnetising inductance; $\left|i_{m s}\right|$ is the magnitude of the stator magnetising current.

It is clear from (2) that the magnitude of the stator flux vector can be obtained by integrating the estimated value of $e_{s x}$. It is known from literature (for example, [3]) that a number of problems are associated with such integration. These problems include saturation, zero drift and potentially a stability problem (a pure integrator in this case would result in a marginally stable system).

The NFO strategy suggests to exclude the flux integrator and to assume that $\left|\psi_{s}\right|$ equals to its reference value $\left|\psi_{s}^{*}\right|$. Then the angular velocity $\omega_{m s}$ is obtained from (3) as $e_{s y} /\left|\psi_{s}^{*}\right|$, integrating which the angular position of the stator flux vector is calculated.

This approach results not only in a simple implementation but also in a number of other desirable properties. First of all, NFO requires very few machine parameters $\left(R_{s}\right.$ and $L_{m}$ only) and, unlike other voltage model based techniques, has very low sensitivity to the stator resistance error. The latter problem was explored in detail in [4].

Another desirable property of NFO is its ability to implicitly correct the frame alignment errors. While the previously introduced reference frame $(x, y)$ can be called the "true" reference frame, i.e. that aligned with the actual $\underline{\psi}_{S}$ position, we will denote by $(d, q)$ a reference frame aligned with the estimated $\psi_{s}$ position. The $(d, q)$ frame is that used by the control algorithm, i.e. the "control" frame. The implicit correction of frame alignment errors means that as soon as the "control" frame deviates from the "true" frame, an implicit mechanism present in the NFO algorithm would act to compensate for this error and eventually to realign both frames again.

The above property was explained in detail in [5]. It was also found that the frame alignment may become unstable under certain conditions in regeneration. An augmentation to the basic NFO algorithm proposed in [5], [6] improved its performance in regeneration but did not completely fixed the instability problem. 


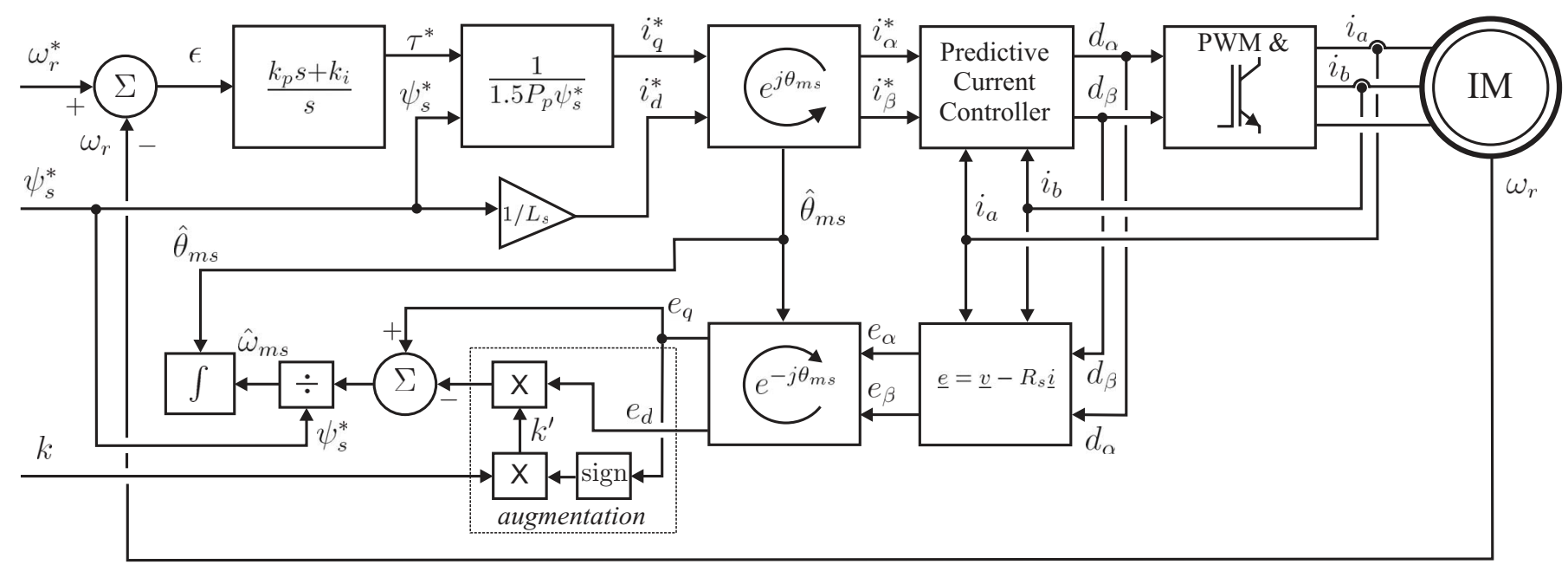

Fig. 1: Block diagram of the NFO algorithm.

In this paper an attempt is made to further improve the NFO performance with respect to its stability in regeneration and under dynamic conditions. The paper has the following structure. Firstly, a dynamic model of the motor-drive system under the NFO control is developed. The linearised transfer functions of the system are then presented and discussed. Based on this discussion, different strategies of the auxiliary feedback implementation are suggested. Their relative advantages and disadvantages are explained and supported by simulation and experimental results. Finally, the conclusions are made about the success and the limitations of the proposed augmentation strategies.

\section{Development of Dynamic Model}

The NFO control algorithm can be explained by means of the block diagram in Fig. 1. In Fig. $1 \alpha, \beta$ are the axes of the stationary reference frame and $d, q$ are the axes of the control frame aligned with the estimated position of the stator flux vector. Conversion the stationary frame to the control frame and vice versa is performed via an estimated angular position $\hat{\theta}_{m s}$ of the stator flux vector, which is obtained by integrating the estimated angular velocity $\hat{\omega}_{m s}$.

The estimation of $\hat{\omega}_{m s}$ is based on equation (3) but uses $e_{s q}$ as an approximation of $e_{s y}$ and the reference flux magnitude $\psi_{s}^{*}$ as an approximation of the actual flux magnitude $\left|\psi_{s}\right|$. The optional augmentation to the basic algorithm shown in Fig. 1 inside a dotted line box was proposed in [5] for stability improvement in regeneration. With the basic and the augmented versions of NFO one has respectively:

$$
\hat{\omega}_{m s}=\frac{e_{s q}}{\psi_{s}^{*}} \quad \text { or } \quad \hat{\omega}_{m s}=\frac{e_{s q}-k^{\prime} e_{s d}}{\psi_{s}^{*}}
$$

where $e_{s d}$ and $e_{s q}$ are projections of the stator back emf vector onto the axes $d$ and $q$ respectively; $k^{\prime}=k \operatorname{sign}\left(e_{s q}\right)$ and $k>0$ is a tunable gain parameter.

In this paper, the reference flux magnitude $\psi_{s}^{*}=$ const. It would be convenient then to normalise the actual stator flux magnitude $\psi_{s}$ by its reference value $\psi_{s}^{*}$. The reference and the actual torque will be normalised by $\tau_{0}^{*}$, where $\tau_{0}^{*}=$ $(3 / 2) P_{p} L_{s}\left(i_{d}^{*}\right)^{2}$ is the reference torque for the case when the reference stator currents are equal, i.e. when $i_{q}^{*}=i_{d}^{*}$. The following main variables will be used to describe the system:

- $x=\tau_{e m}^{*} / \tau_{0}^{*}=i_{q}^{*} / i_{d}^{*}$ is the normalised reference torque or, equally, the ratio of the reference stator currents;

- $y=\psi_{s} / \psi_{s}^{*}=L_{m}\left|i_{m s}\right| / L_{s} i_{d}^{*}$ is the stator flux magnitude normalised by its reference value;

- $\tau_{n}=\tau_{e m} / \tau_{0}^{*}$ is the normalised actual torque;

- $\omega_{r}$ is the mechanical angular velocity of the rotor;

- $\theta_{e}=\hat{\theta}_{m s}-\theta_{m s}$ is the angular error of the control frame, i.e. the difference between the estimated and the true angular positions of the stator flux vector.

With reference to Fig. 1, one can assume that the predictive current controller is very fast and ensures that $i_{\alpha}=i_{\alpha}^{*}$ and $i_{\beta}=i_{\beta}^{*}$ at all times. Neglecting the inverter dead time and nonlinear effects, it can be further assumed that the stator voltages are accurately estimated based on the inverter states. From Fig. 1 it can be seen that only two machine parameters are used by the NFO algorithm, namely, the stator resistance $R_{s}$ and the stator inductance $L_{s}$. The effect of the parameter errors on the system stability was previously explored in [4] and is not included in the scope of this paper, hence the accurate knowledge of these parametes is also assumed.

With the above assumptions the structure of Fig. 1 can be simplified for the purposes of the analysis. Let us assume that, due to calculation errors and disturbances, the estimated position of the stator flux vector differs from its actual position by a small angular error $\theta_{e}$. Then the commanded stator currents $i_{d}^{*}$ and $i_{q}^{*}$ will correspond to the following currents in the "true" reference frame:

$$
\begin{aligned}
& i_{s x}=i_{d}^{*} \cos \theta_{e}-i_{q}^{*} \sin \theta_{e} \\
& i_{s y}=i_{d}^{*} \sin \theta_{e}+i_{q}^{*} \cos \theta_{e}
\end{aligned}
$$


and the stator back emfs estimated in the control frame will differ from those defined in the true frame as per:

$$
\begin{gathered}
e_{s d}=e_{s x} \cos \theta_{e}+e_{s y} \sin \theta_{e} \\
e_{s q}=-e_{s x} \sin \theta_{e}+e_{s y} \cos \theta_{e}
\end{gathered}
$$

For the purposes of the model development we will also use the equations for speed and torque, as well as the rotor voltage equation in the stator flux oriented frame, which can be found, for example, in [3]. The full set of equations describing the dynamic behaviour of the NFO controlled induction machine is given below for convenience:

$$
\begin{aligned}
& J \frac{d \omega_{r}}{d t}=\tau_{e m} ; \quad \tau_{e m}=\frac{3}{2} P_{p} L_{m}\left|i_{m s}\right| i_{s y} \\
& L_{m} \frac{d\left|i_{m s}\right|}{d t}+\frac{1}{T_{r}} L_{m}\left|i_{m s}\right|=\sigma L_{s} \frac{d i_{s x}}{d t}+\frac{1}{T_{r}} L_{s} i_{s x} \\
& \quad-\sigma L_{s} \omega_{s l} i_{s y} \\
& \omega_{s l}=\frac{\sigma L_{s} \frac{d i_{s y}}{d t}+\frac{1}{T_{r}} L_{s} i_{s y}}{L_{m}\left|i_{m s}\right|-L_{s} i_{s x}} ; \quad \frac{d \theta_{e}}{d t}=\omega_{e}=\hat{\omega}_{m s}-\omega_{m s} \\
& \omega_{m s}=P_{p} \omega_{r}+\omega_{s l} ; \quad \hat{\omega}_{m s}=\frac{\hat{e}_{q}-k^{\prime} \hat{e}_{d}}{\psi_{s}^{*}} \\
& i_{s x}=i_{d}^{*} \cos \theta_{e}-i_{q}^{*} \sin \theta_{e} ; \quad i_{s y}=i_{d}^{*} \sin \theta_{e}+i_{q}^{*} \cos \theta_{e} \\
& \frac{d i_{s x}}{d t}=-\left(i_{d}^{*} \sin \theta_{e}+i_{q}^{*} \cos \theta_{e}\right) \frac{d \theta_{e}}{d t}-\sin \theta_{e} \frac{d i_{q}^{*}}{d t} \\
& \frac{d i_{s y}}{d t}=\left(i_{d}^{*} \cos \theta_{e}-i_{q}^{*} \sin \theta_{e}\right) \frac{d \theta_{e}}{d t}+\cos \theta_{e} \frac{d i_{q}^{*}}{d t} \\
& e_{s d}=L_{m} \frac{d\left|i_{m s}\right|}{d t} \cos \theta_{e}+\omega_{m s} L_{m}\left|i_{m s}\right| \sin \theta_{e} \\
& e_{s q}=-L_{m} \frac{d\left|i_{m s}\right|}{d t} \sin \theta_{e}+\omega_{m s} L_{m}\left|i_{m s}\right| \cos \theta_{e}
\end{aligned}
$$

where: $J$ - total inertia of the machine and the load; $T_{r}=$ $L_{r} / R_{r}$ - rotor time constant; $\sigma=1-L_{m}^{2} /\left(L_{s} L_{r}\right)$ - leakage coefficient. Manipulating (9) - (17) and using "dot" notation for the derivatives and the previously introduced normalised variables $x, y$ and $\tau_{n}$, one obtains the following non-linear equations:

$$
\begin{gathered}
\dot{\omega}_{r}=\frac{1}{J} \tau_{n}=\frac{\tau_{0}^{*}}{J} y\left(\sin \theta_{e}+x \cos \theta_{e}\right) \\
\dot{y}+\frac{1}{T_{r}} y=\frac{1}{T_{r}}\left(\cos \theta_{e}-x \sin \theta_{e}\right)-\sigma \dot{\theta}_{e}\left(\sin \theta_{e}+x \cos \theta_{e}\right) \\
\quad-\sigma \dot{x} \sin \theta_{e}-\sigma \omega_{s l}\left(\sin \theta_{e}+x \cos \theta_{e}\right) \\
\dot{\theta}_{e}=-\dot{y}\left(\sin \theta_{e}+k^{\prime} \cos \theta_{e}\right) \\
\quad+\left(P_{p} \omega_{r}+\omega_{s l}\right)\left(y\left(\cos \theta_{e}-k^{\prime} \sin \theta_{e}\right)-1\right)
\end{gathered}
$$

where $\omega_{s l}$ is to be substituted into (19) and (20) from:

$$
\begin{aligned}
& \omega_{s l}= \\
& =\frac{\frac{1}{T_{r}}\left(\sin \theta_{e}+x \cos \theta_{e}\right)+\sigma \dot{\theta}_{e}\left(\cos \theta_{e}-x \sin \theta_{e}\right)+\sigma \dot{x} \cos \theta_{e}}{y-\sigma\left(\cos \theta_{e}-x \sin \theta_{e}\right)}
\end{aligned}
$$

As follows from the physical nature of the variables, (18) is the speed equation; (19) is the flux equation; and (20) is the angular error equation of the plant. These non-linear equations can be written in the following general form:

$$
\begin{aligned}
& \frac{J}{\tau_{0}^{*}} \dot{\omega}_{r}=\tau_{n}=f_{1}\left(x, y, \theta_{e}\right) \\
& \dot{y}=f_{2}\left(\dot{\theta}_{e}, \dot{x}, \theta_{e}, x, y\right) \\
& \dot{\theta}_{e}=\omega_{e}=f_{3}\left(\dot{y}, \dot{x}, y, x, \omega_{r}, \theta_{e}\right)
\end{aligned}
$$

The structure of the control system resulting from expressions (22)-(24) appears in Fig. 2.

A possible modification of the augmented NFO algorithm includes using a PI block instead of the tunable gain in its auxiliary feedback. In that case, the the angular velocity of the control frame will be estimated as:

$$
\hat{\omega}_{m s}=\frac{e_{s q}-k^{\prime} e_{s d}-m^{\prime} \int e_{s d} d t}{\psi_{s}^{*}}
$$

where $k^{\prime}$ and $m^{\prime}$ are, respectively, the proportional and the integral gain that can be controlled in both signs and magnitudes. This version of the augmented NFO algorithm results in that the following equation for $\ddot{\theta}_{e}$ will be used instead of (24):

$$
\ddot{\theta}_{e}=f_{4}\left(\ddot{y}, \ddot{x}, \dot{y}, \dot{x}, \dot{\omega}_{r}, \dot{\theta}_{e}, y, x, \omega_{r}, \theta_{e}\right)
$$

In this case, function $f_{4}$ instead of function $f_{3}$ should be used in the internal non-linear feedback block in the bottom of Fig. 1 .

\section{RESULTS OF THE LINEARISED ANALYSIS}

Two transfer functions are of interest in the following linearised analysis, namely:

- $\delta \tau_{n}(s) / \delta x(s)$ - the transfer function from the normalised reference torque to the normalised actual torque, or the transfer function of the "plant". This transfer function determines the behaviour of the system of Fig. 2 when the PI-controller in the speed loop is saturated and the speed loop is open. The latter situation is typically the case during acceleration, braking or abrupt changes in load;

- $\delta \omega_{r}(s) / \delta \omega_{r}^{*}(s)$ - the transfer function from the reference angular velocity to the actual angular velocity, or the close loop transfer function of the system. This transfer function determines the behaviour of the system when the PI-controller in the speed loop is in the linear mode of operation.

As the details of the linearised analysis itself are not the focus of this paper, here we will only show the results (for the details one can be referred to [7]).

\section{A. Auxiliary feedback of the proportional type}

With this version of the augmented NFO control, the transfer function of the "plant" has the following expression:

$$
\frac{\delta \tau_{n}(s)}{\delta x(s)}=\frac{s\left(a_{2} s^{2}+a_{1} s+a_{0}\right)}{b_{3} s^{3}+b_{2} s^{2}+b_{1} s+b_{0}}
$$

where $a_{0}-a_{2}$ and $b_{0}-b_{3}$ are bulky expressions in terms of the machine parameters and the parameters of the operation point. 


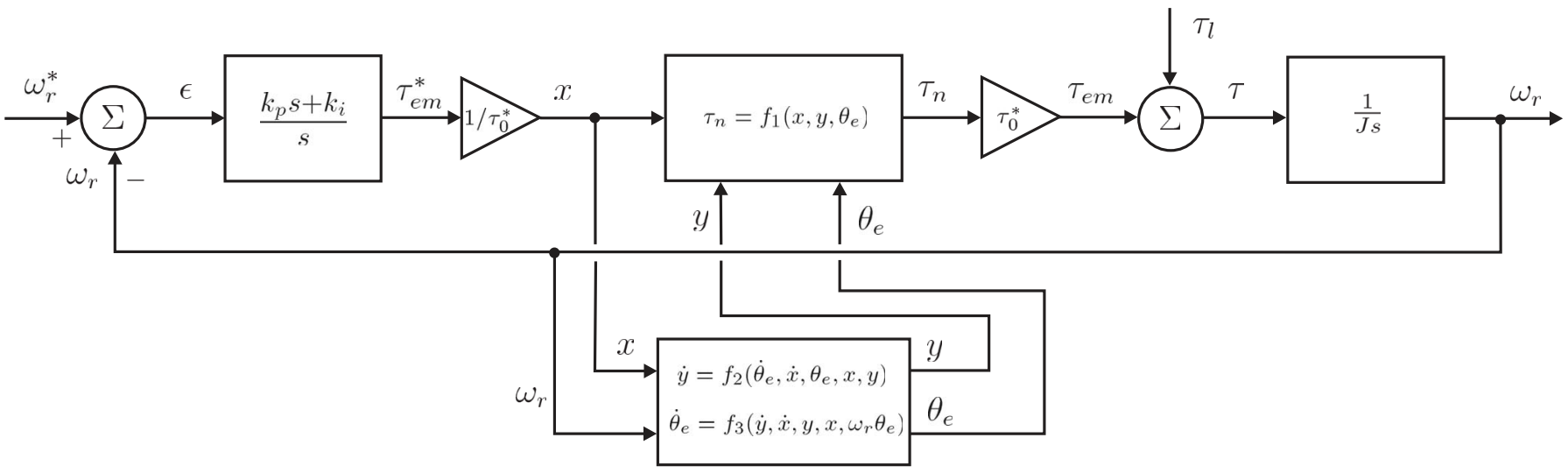

Fig. 2: Block diagram of the non-linear system under the NFO control.

Each of these expressions would take several lines, and their full versions are omitted here for the sake of space. However, by sequentially neglecting the relatively small terms, it is possible to determine, with a reasonable degree of accuracy, the dominant part of each coefficient. Such approximate expressions have been obtained and are given below:

$a_{2} \approx y ; \quad a_{1} \approx y\left(\frac{1}{T_{r}}+\sigma x P_{p} \omega_{r}+k^{\prime} y P_{p} \omega_{r}\right)$

$a_{0} \approx \frac{y}{T_{r}}\left(P_{p} \omega_{r} x-\frac{x^{2}}{y^{2} T_{r}}-\frac{x^{2}}{y T_{r}}-\frac{1}{T_{r}}+k^{\prime} y P_{p} \omega_{r}+k^{\prime} \frac{x}{T_{r}}\right)$

$b_{3} \approx 1 ; \quad b_{2} \approx \frac{1}{T_{r}}+\sigma x P_{p} \omega_{r}+k^{\prime} y P_{p} \omega_{r}$

$b_{1} \approx \frac{1}{T_{r} J}\left(P_{p} \omega_{r} x-\frac{x^{2} \tau_{0}^{*}}{y^{2} T_{r} J}+k^{\prime} y P_{p} \omega_{r}+k^{\prime} \frac{x \tau_{0}^{*}}{T_{r} J}\right)$

$b_{0} \approx \frac{P_{p} \tau_{0}^{*}}{T_{r} J}(y-1)\left(x^{2}-y\right)$

The following observations can be made from examining the expressions (28)-(32):

1) Coefficients $a_{2}$ and $b_{3}$ are always positive;

2) Due to the presence of the $k^{\prime} y P_{p} \omega_{r} y$ term, which is positive in most cases, coefficients $a_{1}$ and $b_{2}$ are also typically positive;

3) Due to the same positive term, coefficients $a_{0}$ and $b_{1}$ can be kept positive under any conditions by using $k^{\prime}$ of the appropriate magnitude;

4) Within the accuracy of this approximation, coefficient $b_{0}$ is independent of the the auxiliary feedback gain $k^{\prime}$ and can take positive or negative values depending on the flux and torque magnitudes.

The close-loop transfer function of the system is defined as:

$$
\begin{aligned}
& \frac{\delta \omega_{r}(s)}{\delta \omega_{r}^{*}(s)}=\frac{\left(k_{p} s+k_{i}\right)\left(a_{2} s^{2}+a_{1} s+a_{0}\right)}{\left(k_{p} s+k_{i}\right)\left(a_{2} s^{2}+a_{1} s+a_{0}\right)+} \\
& \overline{+J s\left(b_{3} s^{3}+b_{2} s^{2}+b_{1} s+b_{0}\right)}
\end{aligned}
$$

\section{B. Auxiliary feedback of the PI type}

For the situation when the PI block is used in the auxiliary feedback, one can obtain the following expression of the transfer function of the "plant":

$$
\frac{\delta \tau_{n}(s)}{\delta x(s)}=\frac{s\left(a_{3} s^{3}+a_{2} s^{2}+a_{1} s+a_{0}\right)}{b_{4} s^{4}+b_{3} s^{3}+b_{2} s^{2}+b_{1} s+b_{0}}
$$

and define the dominant parts of the coefficients $a_{0}-a_{2}$ and $b_{0}-b_{3}$ as follows:

$$
\begin{aligned}
& a_{3} \approx y ; \quad a_{2} \approx P_{p} \omega_{r} k^{\prime} y^{2} \\
& a_{1} \approx-\beta_{8} y+P_{p} \omega_{r} y \frac{x}{T_{r}} \\
& a_{0} \approx \beta_{8} \frac{1}{T_{r}}\left(x \theta_{e}-y\right)+\frac{1}{T_{r}} P_{p} \dot{\omega}_{r} x \\
& b_{4} \approx 1 ; \quad b_{3} \approx P_{p} \omega_{r} y k^{\prime} \\
& b_{2} \approx-\beta_{8}+\frac{P_{p} \omega_{r}}{T_{r}}\left(y k^{\prime}+x\right) \\
& b_{1} \approx-\frac{\beta_{8}}{T_{r}} ; \quad b_{0} \approx-\frac{\tau_{0}^{*}}{T_{r} J} P_{p} y m^{\prime} \sin \theta_{e}\left(x^{2}-y\right) \\
& \beta_{8} \approx-P_{p} y \omega_{r} m^{\prime}-P_{p} y \dot{\omega}_{r} k^{\prime}+x m^{\prime} / T_{r}
\end{aligned}
$$

From examining expressions (35)-(41) one can observe that:

1) Coefficients $a_{3}$ and $b_{4}$ are always positive;

2) Coefficients $a_{2}$ and $b_{3}$ will be positive if $k^{\prime}$ is of the same sign as $\omega_{r}$;

3) The signs of $a_{1}$ and $b_{2}$ will depend on the sign of $\beta_{8}$; $b_{2}$ also includes the component that should be positive if the condition for positive $a_{2}$ and $b_{3}$ is met;

4) Coefficient $a_{0}$ also depends on $\beta_{8}$, however, in the situation when the angular velocity changes, $a_{0}$ will be dominated by the positive second term;

5) Coefficient $b_{1}$ depends on $\beta_{8}$;

6) The sign of coefficient $b_{0}$ depends on the relation between the flux and torque magnitudes, the sign of the angular error and the sign of the proportional gain $m^{\prime}$. 


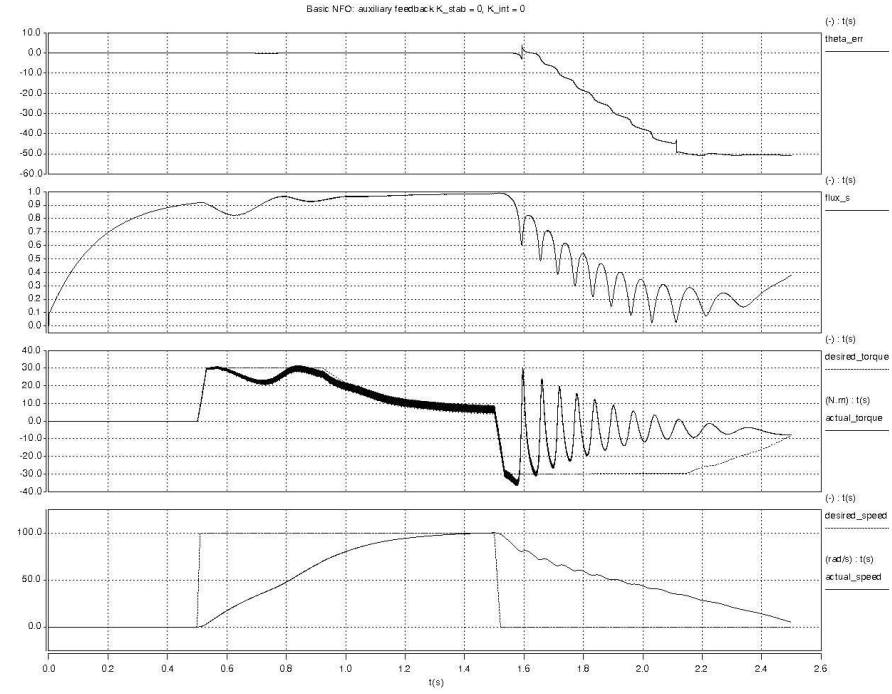

Fig. 3: Simulation results for non-augmented NFO.
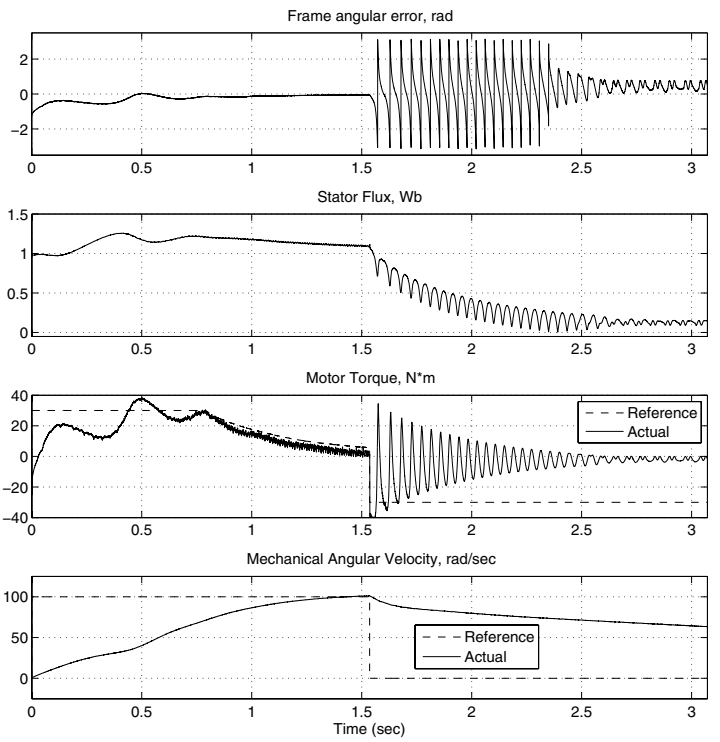

Fig. 4: Experimental results for non-augmented NFO.

The follwing is the expression for the close-loop transfer function of the system:

$$
\begin{aligned}
& \frac{\delta \omega_{r}(s)}{\delta \omega_{r}^{*}(s)}=\frac{\left(a_{3} s^{3}+a_{2} s^{2}+a_{1} s+a_{0}\right)\left(k_{p} s+k_{i}\right)}{\left(a_{3} s^{3}+a_{2} s^{2}+a_{1} s+a_{0}\right)\left(k_{p} s+k_{i}\right)+} \\
& \overline{+J s\left(b_{4} s^{4}+b_{3} s^{3}+b_{2} s^{2}+b_{1} s+b_{0}\right)}
\end{aligned}
$$

\section{DISCUSSION OF DIFFERENT NFO OPTIONS}

Several versions of the NFO control algorithm will be discussed in this section. The discussions are supported by simulations and experimental results obtained for the different NFO

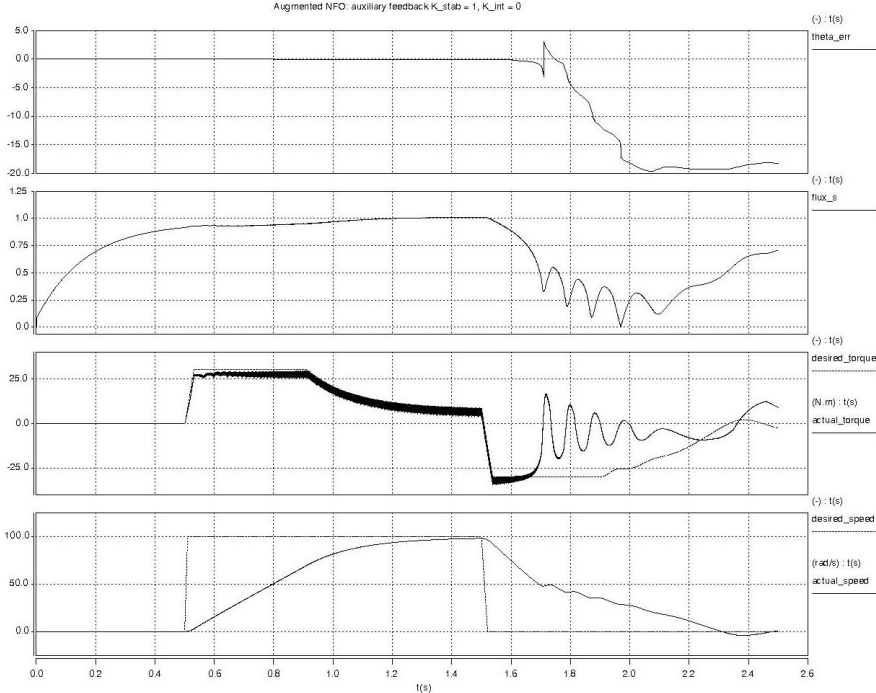

Fig. 5: Simulation results for augmented NFO with $k=1$.

strategies under the same conditions. The machine parameters appear in Table I. The test conditions for both simulations and experiments were as follows: after allowing approximately 0.5 $\mathrm{sec}$ for fluxing, a step change of the reference speed from zero to $100 \mathrm{rad} / \mathrm{sec}(2 / 3$ of the rated speed) was applied to the machine. After bringin it to the commanded speed, a step change back to zero was commanded. These conditions demonstrate performance of the algorithm in both motoring and regeneration. The absolute value of the torque limit is set close to the rated torque of the machine.

The simulations were done for a detailed model of the test machine in Saber ${ }^{\circledR}$ environment. The experiments were carried out using a $38 \mathrm{~kW}$ IGBT based inverter connected to a $7.5 \mathrm{~kW}$ wye connected induction machine. The test machine was mounted on a dynamometer test bed with a DC load machine configured as a simple Ward-Leonard system to provide static loading capable of regeneration or motoring operation.

\section{A. Non-augmented NFO}

We start with the discussion of the basic (non-augmented) NFO control algorithm, which can be regarded as a special case of the augmented NFO when the auxiliary feedback gain $k^{\prime}=0$. Substituting $k^{\prime}=0$ into the expressions (28)-(32) one can see that if $x<0$ then coefficients $a_{0}$ and $b_{1}$ will be typically negative, hence instability of the NFO algorithm in regeneration. This conclusion agrees with the previously reported in ( [5]) and ( [6]) results, and is confirmed by simulation of Fig. 3 and experimental results of Fig. 4. The figures show that the basic NFO algorithm is able to start the machine and to bring it to the commanded speed, but as soon as a negative torque is required the control is lost. At that moment, the speed loop is open as the output of the speed PI controller is limited by its maximum negative value. 

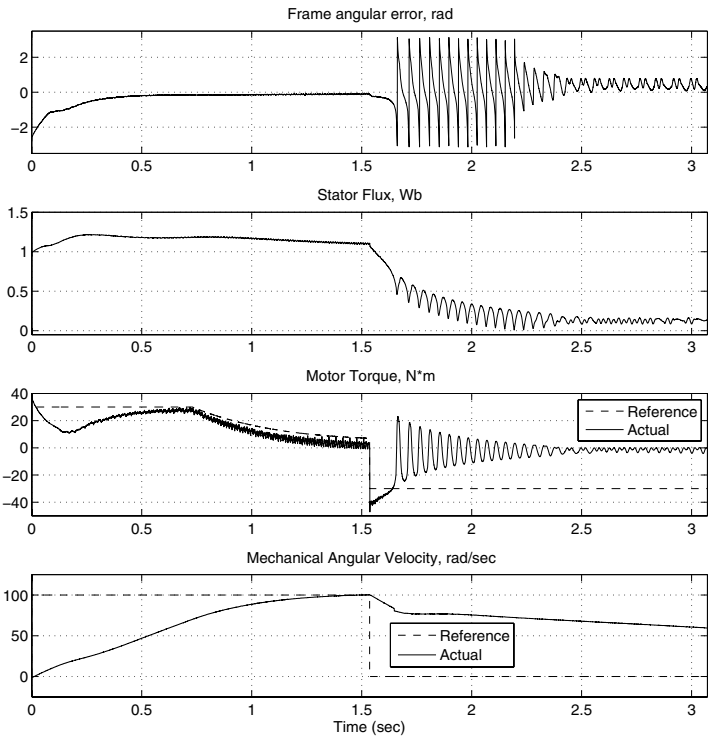

Fig. 6: Experimental results for augmented NFO with $k=1$.

\section{B. Augmented NFO with constant gain in the auxiliary feed- back}

When the auxiliary feedback with constant gain is applied, the algorithm performance improves. By comparing Fig. 5 and Fig. 6 to Fig. 3 and Fig. 4, one can see the ability of the augmented algorithm to track the reference torque in motoring is much better than that of the non-augmented option. When entering regeneration, the augmented algorithm is able to provide negative for some short period of time but then loses control. From the previously reported results (see ( [5]) and ( [6])), the augmented NFO with $k=1$ was capable to stay stable if approximately $-1 / 3$ of the rated torque is applied in steady state or $\mathrm{a}-1 / 6$ of the rated torque is commanded as a step change.

With relation to the expressions (28)-(32), the positive term $k^{\prime} \omega_{r}$ compensates for negative (in regeneration) terms depending on $x$ and provides that $a_{0}>0$ and $b_{1}>0$, to a certain limit of the torque values. However, the uncontrolled coefficient $b_{0}$ changes its sign during the torque reversal, hence the rapid reduction of flux and the rapid increase of the angular error that are observed in Fig. 5 and Fig. 6.

\section{Augmented NFO with flexible gain in the auxiliary feed-} back

Though it follows from the expression (32) that the coefficient $b_{0}$ cannot be directly controlled to guarantee that it is positive, an attempt can be made to keep the conditions such that $b_{0}>0$. On the other hand, if the instable conditions stay only for a short time then the system may be able to quickly re-establish its stability. These possibilities were sought for when we tried to vary the auxiliary feedback gain. The main limitation in this study was to retain the inherent simplicity

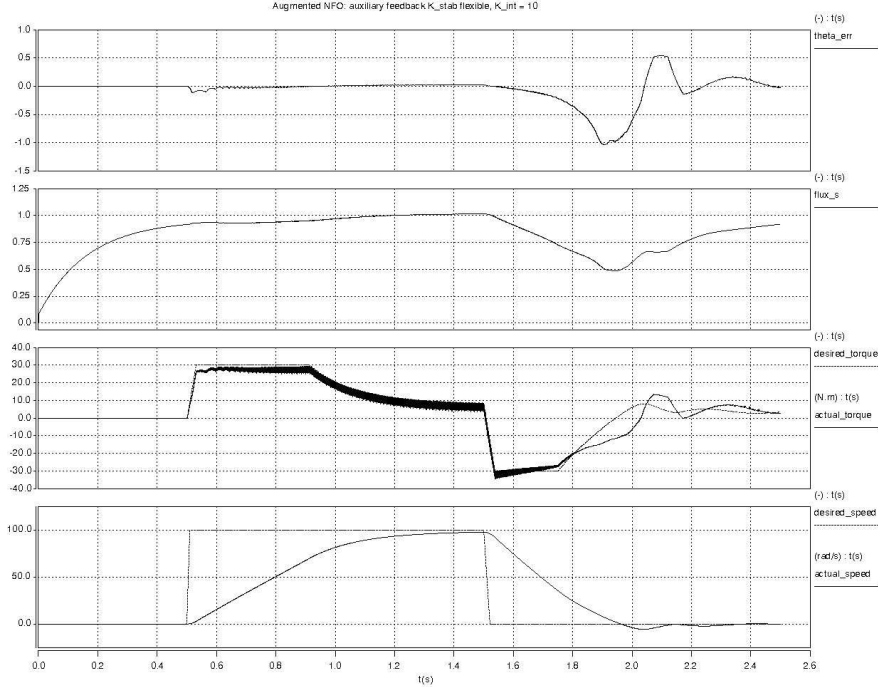

Fig. 7: Simulation results for augmented NFO with flexible gain.
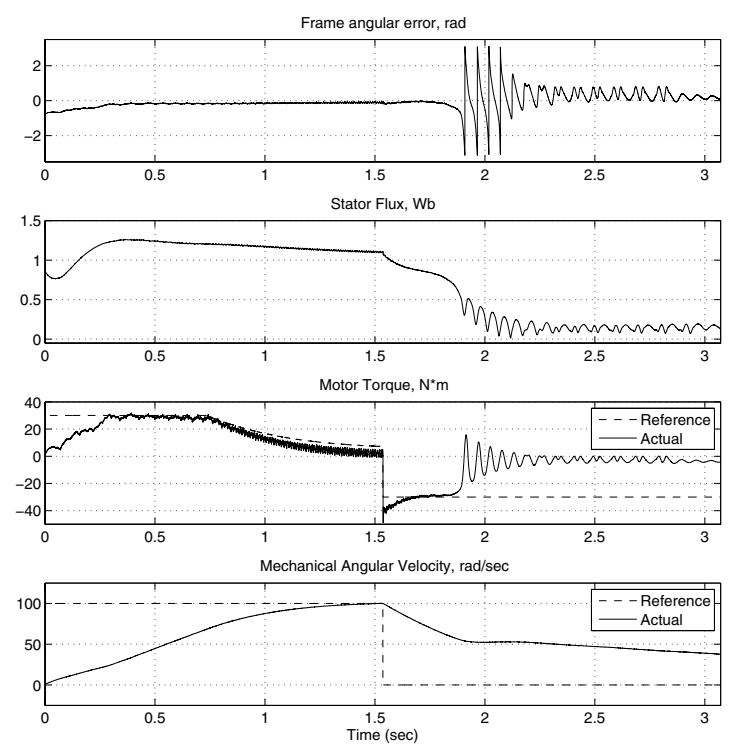

Fig. 8: Experimental results for augmented NFO with flexible gain.

of the NFO algorithm and to use only those parameters for the auxiliary feedback control that NFO is supposed to know, i.e. the commanded speed and torque, $e_{d s}, e_{q s}$ and the rotor speed (measured or estimated).

Different strategies were tried based on the condition as to keep the polynomial coefficients (28)-(32) positive at all times. The best working (so far) strategy - to control the auxiliary feedback gain according to:

$$
k^{\prime}=\operatorname{sign}\left(e_{q}\right)\left(C-x \operatorname{sign}\left(e_{q}\right)\right)
$$

is illustrated by Fig. 7 and Fig. 8. In the simulation of Fig. 7 the machine is successfully started, brought up to speed and then 
back to standstill, though some unstable behaviour is observed as speed approaches zero. This unstable behaviour is much more pronounced in the experimental plots of Fig. 8.

The author has found that when the machine is reversed, the change of sign of the frame angular error would always occur. It can be shown that if the control frame $(d, q)$ is ahead of the "true" frame $(x, y)$, i.e. when $\theta_{e}>0$, then the machine is under-magnetised and $y<1$. In the opposite case, when the control frame is behind the "true" frame, the machine is over-magnetised and $y>1$. This means that during a speed reversal, the coefficient $b_{0}$ from (32) would change its sign and that the NFO algorithm, at least for a short period of time, would lose the control. This is the fundamental limitation of the discussed version of the augmented NFO control.

Another interesting observation on the behaviour of the NFO control with the tunable gain is illustrated by simulation plots of Fig. 11. It can be seen from Fig. 11 that after a negative step of the angular velocity was commanded, instead of slowing down and reversing, the machine further accelerated in the positive direction! This agrees with some reported cases of peculiar behaviour of the NFO-driven golf carts, which was one of the author's original motivations to undertake a study into the stability issues of the NFO control algorithm.

With relation to Fig. 11, after the gain $k^{\prime}$ changed its sign, the angular error $\theta_{e}$ started growing and reached $90^{\circ}$ at which level it settled. In other words, the control frame $(d, q)$ was rotating with the $90^{\circ}$ shift relative to the actual frame $(x, y)$. The estimated angular velocity in equation (4) was growing in proportion to $e_{d s}$ rather than $e_{q s}$ (as it normally should) and the machine was operating in a simingly "controlled" manner while accelerating instead of decelerating.

Similar situations were observed in experiments when trying different versions of the flexible gain control. This peculiar behaviour results from the "symmetry" of the angular velocity estimation given by the expression (4) with $k^{\prime} \neq 0$, with relation to the $e_{d s}$ and $e_{q s}$ variables, so that a possibility exists that, if the frame angular error becomes high, they can swap their roles.

\section{Augmented NFO with the PI block in the auxiliary feed- back}

The above mentioned possibility is excluded when the PI block is used in the auxiliary feedback, with independent control over the signs and the magnitudes of the proportional $k^{\prime}$ and the integral $m^{\prime}$ gains. The logic for such control follows from examining the conditions (35)-(41). To have $a_{2}>0$ and $b_{3}>0$ it is important that $k^{\prime}$ has the same sign as $\omega_{r}$ (more precisely, the same sign as $\left.\omega_{m s}\right)$. The signs of $a_{1}, a_{0}, b_{2}$ and $b_{1}$ depend on the coefficient $\beta_{8}$, which in its turn depends on $m^{\prime}$. It is required that $\beta_{8}<0$.

When $x$ and $\omega_{r}$ are both positive (e.g. acceleration in the positive direction) then, according to the expression (41), $\mathrm{m}^{\prime}$ should be positive but not high (can be even zero), otherwise due to the (positive) third term in (41) $\beta_{8}$ can turn positive. When braking in the positive direction $\left(x<0, \omega_{r}>0\right)$ then $m^{\prime}$ should be positive and high, to compensate for a (positive)

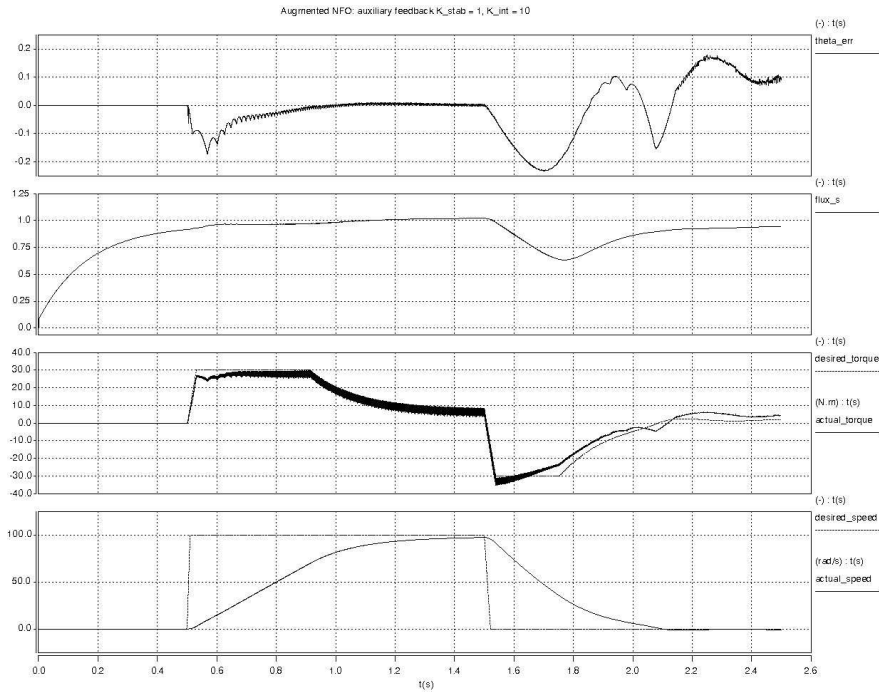

Fig. 9: Simulation results for augmented NFO with PI block.
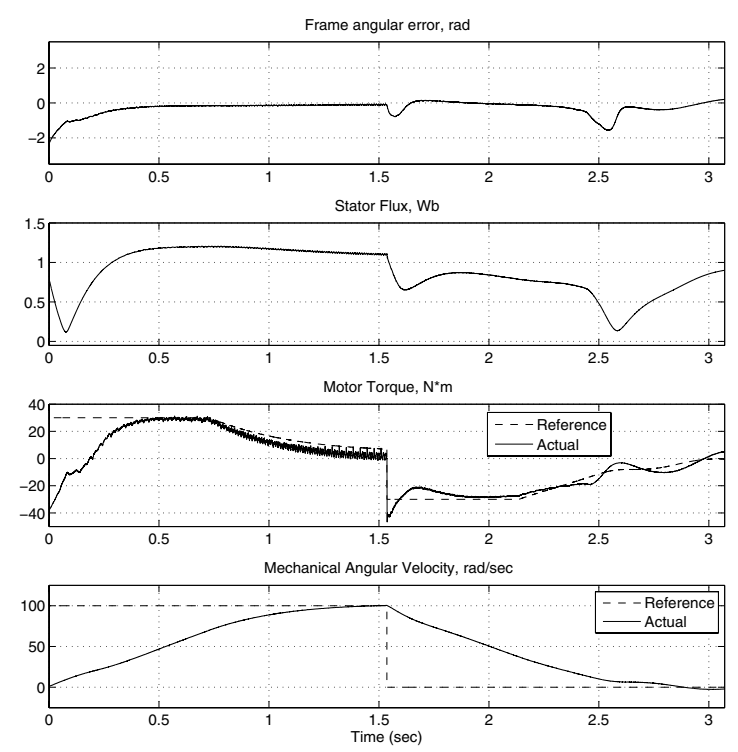

Fig. 10: Experimental results for augmented NFO with PI block.

second term in (41). Similarly, when the machine rotates in the negative direction, then a small negative (or zero) $\mathrm{m}^{\prime}$ value would not prevent from acceleration and a high negative $m^{\prime}$ would assist in braking.

A control strategy based on the above reasoning is illustrated by Fig. 9 and Fig. 10. The $k^{\prime}$ value is controlled in a similar manner to the case discussed in subsection I-B. The $m^{\prime}$ value of the appropriate sign turns on only during regeneration. The resulting performance of the NFO algorithm is the best so far achieved by the author. The simulation plots of Fig. 9 and the experimental plots of Fig. 10 show that the machine was successfully brought up to speed and then returned to 
TABLE I: Parameters of the experimental induction machine.

\begin{tabular}{|l|c|}
\hline \multicolumn{1}{|c|}{ PARAMETER } & VALUE \\
\hline \hline MAGNETISING INDUCTANCE $L_{m}$ & $0.0961 \mathrm{H}$ \\
\hline LEAKAGE INDUCTANCES $L_{l s}, L_{l r}$ & $0.003 \mathrm{H}$ \\
\hline ROTOR RESISTANCE $R_{r}$ & $0.6 \Omega$ \\
\hline STATOR RESISTANCE $R_{s}$ & $0.6 \Omega$ \\
\hline POPLE PAIRS $P_{p}$ & 2 \\
\hline
\end{tabular}

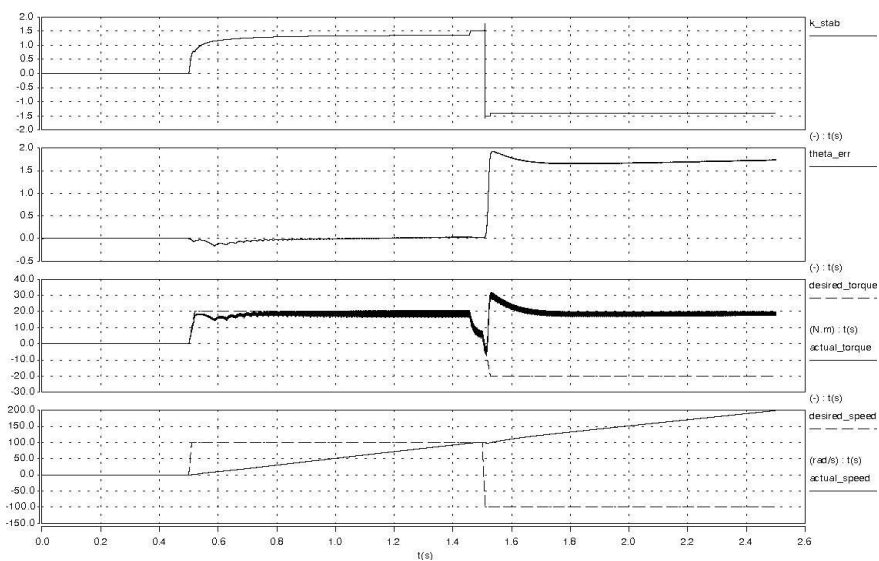

Fig. 11: Simulation of a particular situation with flexible k

standstill. Unfortunately, due to the lack of control over the sign of $b_{0}$, periods of unstable behaviour are observed in these plots too.

\section{CONCLUSIONS AND CONTRIBUTIONS}

It can be concluded from the paper that:

- The performance of the basic NFO algorithm, as well as of the NFO with the constant gain in the auxiliary feedback, do not satisfy the requirements of applications that include periods of regeneration and abrupt changes of the torque;

- The performance of the NFO control algorithm with the tunable gain in the auxiliary feedback could be prone to occasionally starting in the wrong direction;

- The NFO control performance can be substantially improved when applying the auxiliary feedback of the proportional-integral type in the angular velocity estimation;

- Even in the last case, the dynamic performance of the NFO algorithm can be improved only to a certain extent due to the fundamental limitation discussed in this paper;

- In the opinion of the authors, to overcome these limitations it would be needed to include some additional knowledge of the operation variables and the machine parameters, which would compromise the inherent simplicity of the NFO algorithm;

- The theoretical conclusions, results of simulations and experiments presented in this paper agree with the previously reported results and provide the grounds for their fundamental understanding.

The following are the main contributions of the paper:
- The dynamic model of the drive-motor system controlled by the NFO algorithm has been developed.

- The results of the linearised stability analysis for the NFO algorithm augmented by a proportional and a proportional-and- integral type of the auxiliary feedback have been presented.

- The stability problems of the NFO algorithm have been identified and related to the parameters of the dynamic model.

- Based on the above, strategies for the control of the auxiliary feedback parameters have been suggested.

- As the result of these strategies, the performance of the NFO algorithm, particularly, in regeneration has been substantially improved.

- The fundamental limitations of the NFO control algorithm have been explored.

- The main conclusions of the paper have been backed up by the simulations and experimental results obtained for the test induction machine.

Another possible approach to the remaining instability problems is the improvement of the slow speed performance of the NFO algorithm suggested in [8].

\section{REFERENCES}

[1] R. Jönsson, "Method and apparatus for controlling an AC motor," United States Patent 4,458,193, July 1984.

[2] R. Jönsson, "Method and apparatus for controlling an ac induction motor by indirect measurement of the air-gap voltage," United States Patent 5,294,876, March 1994.

[3] P. Vas, Sensorless Vector and Direct Torque Control. Oxford University Press, 2003.

[4] G. Mirzaeva and R. E. Betz, "Parameter sensitivity issues in natural field orientation," in Industry Applications Conference, 2007. 42nd IAS Annual Meeting. Conference Record of the 2007 IEEE, New Orleans, LA, Sept. 23-27, 2007.

[5] R. E. Betz and G. Mirzaeva, "Frame alignment stability issues in natural field orientation," in Industry Applications Conference, 2006. 41st IAS Annual Meeting. Conference Record of the 2006 IEEE, vol. 5, Tampa, FL, Oct. 8-12, 2006, pp. 2411-2418.

[6] G. Mirzaeva and R. E. Betz, "An improved natural field orientation control of a current fed induction machine," in CDROM Proceedings of IEEEIEMDC 2007, Antalya, Turkey, May 2007.

[7] G. Mirzaeva and A. Rojas, "Stability analysis of the natural field orientation controlled induction machine," in Accepted for presentation at EPE-PEMC 2008, Posnan, Poland, September 2008.

[8] G. Mirzaeva and R. E. Betz, "Slow speed performance of an nfocontrolled induction machine," in Australasian Universities Power Engineering Conference, 2007. CDROM Proceedings of the AUPEC2007, Perth, WA, Dec. 9-12, 2007. 